\title{
ECG De-noising using Hybrid Linearization Method
}

\author{
P Sri Lakshmi ${ }^{*}$ V Lokesh Raju \\ Department of Electronics and Communication Engineering, \\ Aditya Institute of Technology and Management (AITAM), Tekkali \\ *Corresponding author, e-mail: hymap23@gmail.com
}

\begin{abstract}
Electrocardiogram (ECG) is a non-invasive tool that monitors the electrical activity of the heart. An ECG signal is highly prone to the disturbances such as noise contamination, artifacts and other signals interference. So, an ECG signal has to be de-noised so that the distortions can be eliminated from the original signal for the perfect diagnosing of the condition and performance of the heart. Extended Kalman Filter (EKF) de-noises an ECG signal to some extent. This project proposes a method called Hybrid Linearization Method which is a combination of Extended Kalman Filter along with Discrete Wavelet Transform (DWT) resulting in an improved de-noised signal.
\end{abstract}

Keywords: electrocardiograph, extended Kalman filter, discrete wavelet transform

Copyright $\odot 2015$ Institute of Advanced Engineering and Science. All rights reserved.

\section{Introduction}

Electrocardiography is the process of recording the electrical activity of the heart. The Voltage versus Time graph produced by this non invasive medical procedure is referred to as an electrocardiogram which is a time varying signal [1].

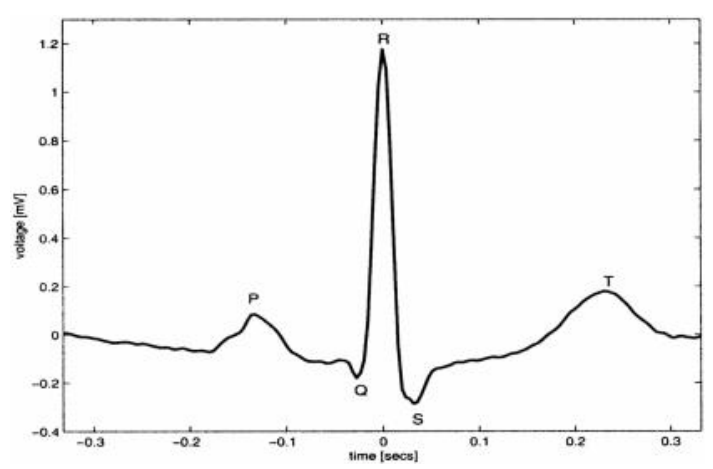

Figure 1. Morphology of a mean PQRST-complex of an ECG recorded from a normal human

A single normal cycle of an ECG waveform reflects the peaks and troughs labeled $P, Q$, $R, S$ and $T$. An ECG signal may sometimes heavily mask by the noise so that the presence of the signal of interest may not be detected. Extracting useful clinical information from the real ECG requires R-peak detection [2] and QT-interval detection [4]. So, an ECG signal must be denoised to estimate the condition of the heart. An ECG signal can be de-noised using Extended Kalman Filter (EKF) [5-6] which can extract the signal from the noise to some extent.

\section{Proposed Method}

The useful information in an ECG signal is observed from the intervals and amplitude of the signal. To estimate the condition of the heart, an ECG signal must be extracted from the noise i.e., an ECG signal must be de-noised. Extended Kalman Filter (EKF) de-noises the signal to some extent. To improve the quality of de-noising, Hybrid Linearization Method is proposed in 
this paper which is a combination of Extended Kalman Filter followed by Discrete Wavelet Transform (DWT) [3] implemented using wavelet toolbox [7].

\subsection{Extended Kalman Filter}

The EKF is a non linear extension of conventional Kalman filter that has been specifically developed for systems having non linear dynamic model. A discrete and non linear system $x_{k+1}=f\left(x_{k}, w_{k}\right)$ and its non linear observation $y_{k}=g\left(x_{k}, v_{k}\right)$ can be approximated linearly near reference point $\left(\begin{array}{lll}\Lambda & \Lambda & \Lambda \\ x_{k}, w_{k}, v_{k}\end{array}\right)$ as follows:

$$
\left\{\begin{array}{c}
x_{k+1}=f\left(\begin{array}{cc}
\Lambda & \Lambda \\
x_{k}, w_{k}
\end{array}\right)+A_{k}\left(\begin{array}{c}
\Lambda \\
x_{k}-x_{k}
\end{array}\right)+F_{k}\left(\begin{array}{c}
\Lambda \\
w_{k}-w_{k}
\end{array}\right) \\
y_{k}=g\left(\begin{array}{ll}
\Lambda & \Lambda \\
x_{k}, v_{k}
\end{array}\right)+C_{k}\left(\begin{array}{c}
\Lambda \\
x_{k}-x_{k}
\end{array}\right)+G_{k}\left(\begin{array}{c}
\Lambda \\
v_{k}-v_{k}
\end{array}\right)
\end{array}\right.
$$

Where $x_{k}$ the state vector, $w_{k}$ the state noise with zero mean and covariance matrices $Q_{k}=E\left\{w_{k} w_{k}^{T}\right\}, f(\bullet)$ the non linear dynamic, $y_{k}$ observation vector, $v_{k}$ the measurement noise with zero mean and covariance matrices $R_{k}=E\left\{v_{k} v_{k}^{T}\right\}, g(\bullet)$ the non linear sensor model and $A_{k}, F_{k}, C_{k}, G_{k}$ are the jacobian matrices given by:

$$
\begin{aligned}
& A_{k}=\left.\frac{\partial f\left(x, \hat{w}_{k}\right)}{\partial x}\right|_{\substack{\wedge \\
x_{k}=x_{k}}} \\
& F_{k}=\left.\frac{\partial f\left(\begin{array}{l}
\Lambda \\
x_{k}, w_{k}
\end{array}\right)}{\partial w}\right|_{\substack{\hat{w}_{k}=w_{k} \\
\text {. }}} \\
& C_{k}=\left.\frac{\partial g\left(x, \hat{v}_{k}\right)}{\partial x}\right|_{x_{k}=x_{k}} \\
& G_{k}=\left.\frac{\partial g\left(\begin{array}{l}
\Lambda \\
\left.x_{k}, w_{k}\right)
\end{array}\right.}{\partial v}\right|_{\substack{v_{k}=v_{k} \\
\text {. }}}
\end{aligned}
$$

Then, extended Kalman equations can be given by:

$$
\begin{aligned}
& \hat{x}_{k / k-1}=f\left(x_{k-1 / k-1}^{\wedge}, 0\right) \\
& P_{k / k-1}=A_{k} P_{k-1 / k-1} A_{k}^{T}+F_{k} Q_{k} F_{k}^{T} \\
& \hat{x}_{k / k}=x_{k / k-1}+K_{k}\left[y_{k}-g\left(\hat{x}_{k / k-1}, 0\right)\right] \\
& K_{k}=P_{k / k-1} C_{k}^{T}\left[C_{k} P_{k / k-1} C_{k}^{T}+G_{k}^{T}\right]^{-1} \\
& P_{k / k}=P_{k / k-1}-K_{k} C_{k} P_{k / k-1}
\end{aligned}
$$


Where ${ }^{\Lambda} x_{k / k-1}=E\left\{x_{k} \mid y_{k-1}, y_{k-2}, \ldots \ldots, y_{1}\right\}$, is estimate of the state vector $x_{k}$ at instant $k$ given observations $y_{1}$ to $y_{k-1}$, and $x_{k / k}=E\left\{x_{k} \mid y_{k}, y_{k-1}, \ldots \ldots, y_{1}\right\}$ is estimate of the state vector $x_{k}$ at instant at instant $k$ given observations $y_{1}$ to $y_{k} \cdot P_{k / k-1}$ and $P_{k / k}$ are defined in the same manner.

\subsection{Discrete Wavelet Transform}

A wavelet is a mathematical function used to divide a function or continuous time signal into different scale components assigning frequency range to each scale component. Discrete wavelet transforms are applied to discrete data sets which maps data from the time domain to the wavelet domain. It has its own advantages over Fourier transform in representing the functions that have discontinuities and sharp peaks for accurately deconstructing and reconstructing finite, non-periodic and non-stationary signals. The forward DWT coefficients for a sequence $f(n)$ are:

$$
\begin{aligned}
& W_{\varphi}\left(j_{0}, k\right)=\frac{1}{\sqrt{M}} \sum_{n} f(n) \varphi_{j_{0}, k}(n) \\
& W_{\psi}(j, k)=\frac{1}{\sqrt{M}} \sum_{n} f(n) \psi_{j, k}(n) \text { for } j \geq j_{0}
\end{aligned}
$$

And the complementary inverse DWT is:

$$
f(n)=\frac{1}{\sqrt{M}} \sum_{k} W_{\varphi}\left(j_{0}, k\right) \varphi_{j_{0}, k}(n)+\frac{1}{\sqrt{M}} \sum_{j=j_{0}}^{\infty} \sum_{k} W_{\psi}(j, k) \psi_{j, k}(n)
$$

The DWT represents the temporal features of a signal at different resolutions, suitable to analyze the ECG signals characterized by a cyclic occurrence of patterns with different frequency content. The DWT is implemented on ECG signal using Wavelet toolbox. The wavelet toolbox is a collection of functions built on the Matlab technical computing environment which provides tools for the analysis and synthesis of signals and images using wavelets and wavelet packets within the framework of Matlab. The toolbox uses graphical interactive tools which is a collection of graphical interface tools that afford access to excessive functionality.

\subsection{Description of the Algorithm}

The algorithm used to de-noise an ECG signal is an efficient algorithm which uses Hybrid Linearization Method - a combination of Extended Kalman Filter (EKF) and Discrete Wavelet Transform (DWT). It has the following procedure of de-noising an ECG signal: first an ECG signal is loaded and Extended Kalman Filter is applied to it. The EKF linearize and denoises the ECG signal to some extent. Root mean square error is calculated from the output of the EKF. Then the signal is applied to the Discrete Wavelet Transform using wavelet toolbox and then the output is calculated. The results from both EKF and DWT can be compared.

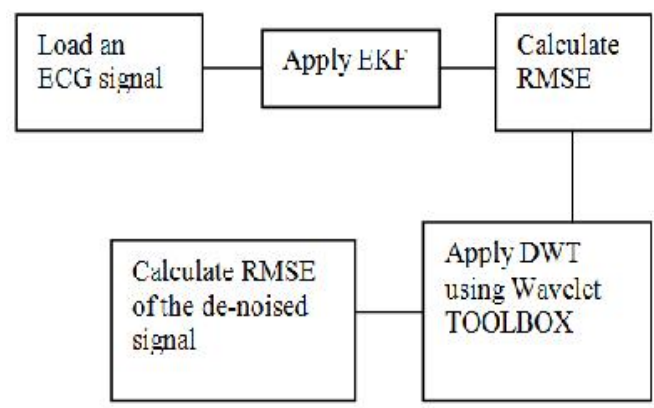

Figure 2. Describing the algorithm to de-noise and ECG signal using Hybrid Linearization Method 
The steps of the algorithm are as follows:

STEP-1: Load the ECG signal

STEP-2: Apply Extended Kalman Filter

STEP-3: Calculate RMSE

STEP-4: Apply the output of EKF to Discrete Wavelet Transform using Wavelet toolbox

STEP-5: Calculate the RMSE of the de-noised signal

STEP-6: Compare the results of steps 3 and 5.

\section{Results}

The experimental study of both the Extended Kalman Filter and Hybrid Linearization Technique which is a combination of EKF and Discrete Wavelet Transform is carried out to evaluate the performance of the proposed method. Let an ECG signal which is a combination of both original signal with noise which is to be applied to the Extended Kalman Filter as shown in the Figure 3:

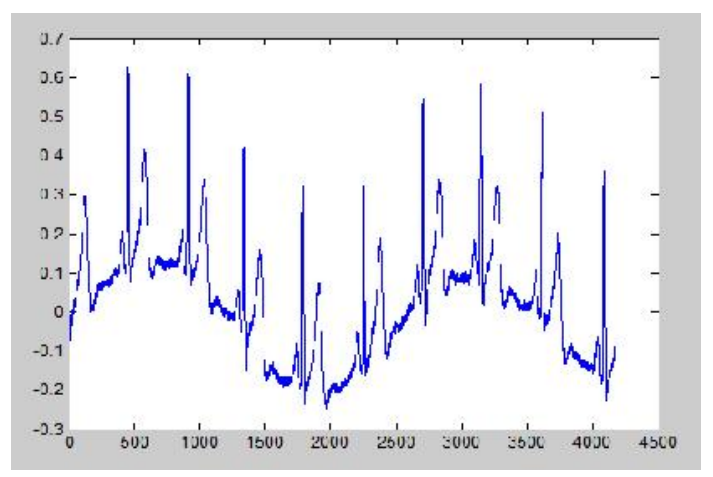

Figure 3. An ECG signal

When an ECG signal is applied to the Extended Kalman Filter the signal is de-noised to some extent and its root mean square error is $4.1322 \mathrm{e}-004$ as shown in Figure 4:

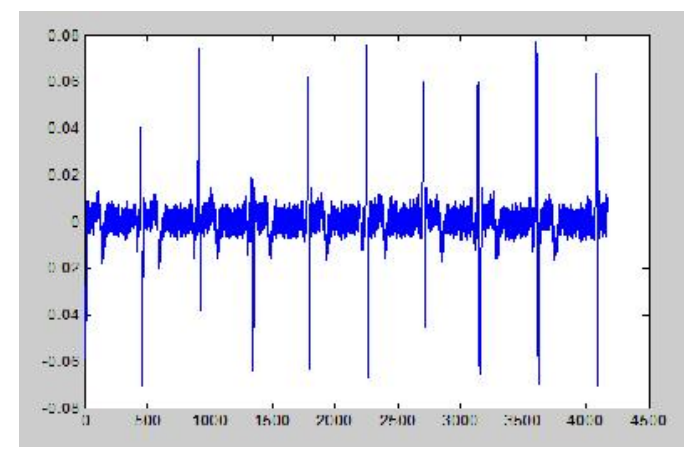

Figure 4. An ECG signal after de-noising with Extended Kalman Filter

The ECG signal extracted from the Extended Kalman Filter is applied to the Discrete Wavelet Transform which is nothing but Hybrid Linearization Method. Thus the Hybrid Linearization Method de-noises the signal improving the root mean square error to $3.1771 \mathrm{e}-004$ as shown in Figure 5: 


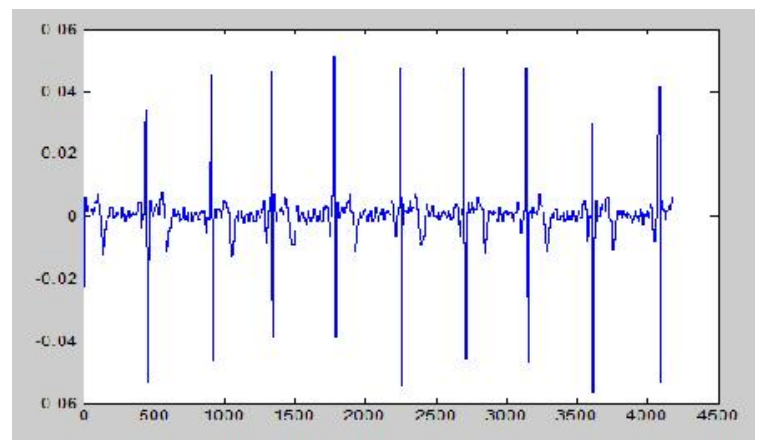

Figure 5. An ECG signal after de-noising using Hybrid Linearization Method

The results extracted from both the Extended Kalman Filter and Hybrid Linearization Method are tabularized in Table 1 through which it approves that the proposed method minimizes the effect of noise on the ECG signal.

Table 1. Performance results for ECG De-Noising

\begin{tabular}{cccc}
\hline $\begin{array}{c}\text { De-Noising using Extended Kalman Filter only } \\
\text { Sum squares }\end{array}$ & $\begin{array}{c}\text { De-Noising using Hybrid Linearization Method } \\
\text { RMSE }\end{array}$ & Sum squares & RMSE \\
\hline $1.7075 \mathrm{e}-007$ & $4.1322 \mathrm{e}-004$ & $1.0094 \mathrm{e}-007$ & $3.1771 \mathrm{e}-004$ \\
\hline
\end{tabular}

\section{Conclusion}

Extended Kalman Filter removes the noise from an ECG signal by linearizing it. This process removes the noise to some extent. This paper proposes Hybrid Linearization Method, a combination of Extended Kalman Filter along with Discrete Wavelet Transform for the improvement of the de-noising of an ECG signal. The results of this paper prove the applicability of the Hybrid Linearization Method to de-noise the ECG signals.

\section{References}

[1] Patrick E McSharry, Gari D Clifford, Lionel Tarassenko, Leonard A Smith. A dynamical model for generating synthetic electrocardiogram signals. IEEE Transactions on Biomedical Engineering. 2003; $50(3)$.

[2] J Pan, WJ Tompkins. A real-time QRS-detection algorithm. IEEE Trans. Biomed. Eng. 1985; BME-32: 220-236.

[3] Channappa Bhyri, Kalpana V, ST Hamde, LM Waghmare. Estimation of ECG features using LabView. TECHNIA- International Journal of Computing Science and Communication Technologies. 2009; 2(1).

[4] P Davey. A new physiological method for heart rate correction of the QT-interval. In Heart. 1999; 82: 183-186.

[5] MS Grewal, AP Andrews. Kalman Filtering: Theory and Practice Using Matlab®. 2nd Edition. John Wiley \& Sons Inc. 2001.

[6] Mohammed Assam Ouali, K Chafaa, Mouna Ghanai, LM Lorente. ECG de-noising using Extended Kalman filter. IEEE. 2013.

[7] M Misiti, Y Misiti. Wavelet Toolbox for using with Matlab. The Math Works. 1996. 